\title{
THE MOCK MODULAR DATA OF A FAMILY OF SUPERALGEBRAS
}

\author{
CLAUDIA ALFES AND THOMAS CREUTZIG
}

(Communicated by Ken Ono)

\begin{abstract}
The modular properties of characters of representations of a family of $\mathrm{W}$-superalgebras extending $\widehat{\mathfrak{g l}}(1 \mid 1)$ are considered. Modules fall into two classes, the generic type and the non-generic one. Characters of non-generic modules are expressed in terms of higher-level Appell-Lerch sums. We compute the modular transformations of characters and interpret the Mordell integral as an integral over characters of generic representations. The $\mathbb{C}$-span of a finite number of non-generic characters together with an uncountable set of characters of the generic type combine into a representation of $\mathrm{SL}(2 ; Z)$. The modular transformations are then used to define a product on the space of characters. The fusion rules of the extended algebras are partially inherited from the known fusion rules for modules of $\widehat{\mathfrak{g l}}(1 \mid 1)$. Moreover, the product obtained from the modular transformations coincides with the product of the Grothendieck ring of characters if and only if the fusion multiplicities are at most one.
\end{abstract}

\section{INTRODUCTION}

Automorphic forms, representation theory and conformal field theory are closely related areas since characters of modules of conformal field theories possess modular properties. Important examples are representations of affine Lie algebras and their characters as well as lattice vertex operator algebras whose characters involve the theta function of the lattice. A crucial ingredient of the algebraic structure of (rational) conformal field theories is the fusion ring of modules, which is related to the modular transformations of characters via the Verlinde formula.

Here, we consider a family of W-superalgebras extending $\widehat{\mathfrak{g l}}(1 \mid 1)$, which is a supersymmetric analogue of $\widehat{\mathfrak{g l}}(1)$, and rank one lattice vertex algebras. To illustrate the main ideas of this construction, the investigation of modularity, and the Verlinde formula we start by presenting the well-known example of rank one lattice vertex algebras. For an introduction to conformal field theory and a detailed exposition of this example, see $G$. The affine vertex algebra of $\mathfrak{g l}(1)$ or rank one Heisenberg algebra has the inverse of Dedekind's $\eta$-function as its character. This algebra admits a family of extensions to larger conformal field theories as follows. Irreducible modules $V_{\alpha}$ of highest-weight $\alpha \in \mathbb{R}$ possess rather trivial fusion products, namely

$$
V_{\alpha} \times_{f} V_{\beta}=V_{\alpha+\beta} .
$$

Received by the editors July 19, 2012.

2010 Mathematics Subject Classification. Primary 11F22, 11F37, 81T40. 
This implies that the module

$$
M_{\alpha}=\bigoplus_{m \in \mathbb{Z}} V_{m \alpha}
$$

is closed under the fusion product. Let $\alpha^{2} \in \mathbb{Z}$; then such a module extends the rank one Heisenberg algebra to a larger conformal field theory. Important examples of these extensions are the affine vertex algebra of $\mathfrak{s l}(2)$ at level one $(\alpha=\sqrt{2})$ and the super Virasoro algebra at central charge one $(\alpha=\sqrt{3})$. The character of the module $V_{\alpha}$ is

$$
\chi_{V_{\alpha}}(u ; \tau)=\frac{e^{2 \pi i \alpha u} e^{\pi i \tau \alpha^{2}}}{\eta(\tau)}
$$

where $\tau \in \mathbb{H}, u \in \mathbb{C}$. Hence, the character of the module $M_{\alpha}$ is

$$
\chi_{M_{\alpha}}(u ; \tau)=\frac{1}{\eta(\tau)} \sum_{m \in \mathbb{Z}} e^{2 \pi i \alpha m u} e^{\pi i \tau \alpha^{2} m^{2}}=\frac{\theta_{3}\left(\alpha u ; \alpha^{2} \tau\right)}{\eta(\tau)} .
$$

If $\alpha$ is the square root of a positive even integer, these characters have particularly good modular properties. The extended algebra is the lattice $\alpha \mathbb{Z}$. The modules

$$
M_{\alpha, n}=\bigoplus_{m \in \mathbb{Z}} V_{m \alpha+n / \alpha}
$$

for $n=0, \ldots, \alpha^{2}-1$ close under the fusion product, and its characters form a representation of the modular group $\mathrm{SL}(2 ; \mathbb{Z})$.

Here we investigate a supersymmetric analogue of this simple example. Consider the affine Lie superalgebra $\widehat{\mathfrak{g l}}(1 \mid 1)$. The character of its affine vertex superalgebra is

$$
\chi_{\widehat{\mathfrak{g}}(1 \mid 1)}(u, v ; \tau)=i \frac{e^{i \pi u}}{1-e^{2 \pi i u}} \frac{\theta_{1}(u ; \tau)}{\eta^{3}(\tau)},
$$

where $\tau \in \mathbb{H}, u, v \in \mathbb{C}$ and $\theta_{1}(u ; \tau)=-i \sum_{n \in \mathbb{Z}}(-1)^{n} e^{\pi i\left(n+\frac{1}{2}\right)^{2} \tau+2 \pi i u\left(n+\frac{1}{2}\right)}$. Verma modules of this algebra are generically irreducible. The non-generic case is called atypical and gives rise to interesting extended algebras [CR1. Atypical modules are labeled by a pair $(n, \ell)$, where $\ell$ is an integer and $n$ a real number. Their characters are, for example if $\ell>0$,

$$
\chi_{\mathcal{A}_{n, \ell}}(u, v ; \tau)=(-1)^{\ell+1} i e^{2 \pi i v \ell} \frac{e^{\pi i\left(2 n u+\left(2 n \ell+\ell^{2}\right) \tau\right)}}{1-e^{2 \pi i(u n+\ell \tau)}} \frac{\theta_{1}(u ; \tau)}{\eta^{3}(\tau)} .
$$

The fusion product of atypical modules is not complicated [CR1]. As in the $\widehat{\mathfrak{g l}}(1)$ case, there exist sets of modules that close under fusion and thus combine to an extended algebra. We are considering a class that is labeled by two integers $n, \ell$ such that $\ell>0, n \equiv 0(\bmod \ell)$ and $n \ell+\ell^{2}>0$. We call these extended algebras $\mathfrak{W}_{n, \ell}$. The most interesting family in this class is obtained by setting $\ell=1$ and $n=0,1,2, \ldots$ In CR2 it was shown that the operator product algebra of an even subalgebra of $\mathfrak{W}_{n, \ell}$ agrees with the operator product algebra of the FeiginSemikhatov $\mathbf{W}_{2 n+1}^{(2)}$ algebra at level $-2(n-1)(2 n+1) /(2 n-1)[\mathrm{FS}$. The character of such an extended algebra is

$$
\chi_{\mathfrak{W}_{n, \ell}}(u, v ; \tau)=i \frac{\theta_{1}(u ; \tau)}{\eta^{3}(\tau)} \sum_{m \in \mathbb{Z}} \frac{e^{\pi i(m \ell+(2 m n+1) u+2 m v+m \ell(m \ell+2 m n+1) \tau)}}{1-e^{2 \pi i(u+m \ell \tau)}} .
$$


This character can be expressed in terms of a higher-level Appell-Lerch sum. Using the modularity of these sums we find analogously to $\widehat{\mathfrak{g l}}(1)$ and rank one lattice vertex algebras

Theorem 1.1. Let $n, \ell \in \mathbb{Z}$, such that $\ell>0, n \equiv 0(\bmod \ell)$ and $n \ell+\ell^{2}>0$. Then there exists a set of modules of the algebra $\mathfrak{W}_{n, \ell}$ such that the $\mathbb{C}$-span of its characters is a representation of the modular group $\mathrm{SL}(2 ; \mathbb{Z})$.

The set of characters of modules appearing involves a finite subset of non-generic, we call them again atypical, modules whose characters are expressed in terms of Appell-Lerch sums, as well as a set of uncountable size of generic or typical ones. We are able to relate the latter to the shifted Mordell integral appearing in the $S$ transformation (the fractional linear transformation given by the involution $\left(\begin{array}{cc}0 & -1 \\ 1 & 0\end{array}\right)$ of the modular group $\mathrm{SL}_{2}(\mathbb{Z})$ ) of the Appell-Lerch sum thus giving a representation theoretical interpretation of this term. A phenomenon of similar type has been noticed in string theory $[\mathrm{M}, \mathrm{ET}$.

A connection between the representation theory of affine Lie superalgebras, Appell-Lerch sums, and number theory was first noticed by Kac and Wakimoto KW2 (see also [KP, KW1]). They discovered that characters of affine Lie superalgebras at level one are related to Ramanujan's mock theta functions [KW2. Using the work of Zwegers [Z2, Chapter 1] on Appell-Lerch sums and mock theta functions, Bringmann and Ono $[\mathrm{BriO}$ ] were able to show that some of the $q$-series obtained by Kac and Wakimoto are the "holomorphic" parts of some non-holomorphic functions which are products of weight 1/2 harmonic Maass forms (introduced by Bruinier and Funke $[\mathrm{BF}$ ) with a weight $-1 / 2$ quotient of $\eta$-functions. Recently, characters of other Lie superalgebras have been related to Appell-Lerch sums (for example GQS, [F], BriFo]). Zwegers [Z3] also embedded other types of $q$-series, namely those that Kac and Wakimoto referred to as "multivariable Appell functions", in a theory similar to the one he built around the level one Appell-Lerch sum in his doctoral thesis [Z2]. We use his results to obtain the modularity of our characters.

In the second part of this article we investigate the connection of the product induced by the modular transformations of characters to the Grothendieck ring of characters. In rational conformal field theories it is straightforward to compute a product structure on the ring of characters using their modular properties. Namely, in the above example the character of the module $M_{\alpha, n}$ is

$$
\chi_{M_{\alpha, n}}(u ; \tau)=z^{n / \alpha} q^{n^{2} / 2 \alpha^{2}} \frac{1}{\eta(\tau)} \theta_{3}\left(\alpha u+n \tau ; \alpha^{2} \tau\right) .
$$

Then the $S$-transformation is

$$
\chi_{M_{\alpha, n}}\left(\frac{u}{\tau} ;-\frac{1}{\tau}\right)=\frac{1}{\sqrt{\alpha^{2}}} e^{\pi i u^{2} / \tau} \sum_{l=0}^{\alpha^{2}-1} e^{-2 \pi i n l / \alpha^{2}} \chi_{M_{\alpha, l}}(u ; \tau) .
$$

This transformation is used to define the numbers

$$
S_{n l}=\frac{e^{-2 \pi i n l / \alpha^{2}}}{\sqrt{\alpha^{2}}} .
$$


The matrix $S$ with entries $S_{n l}$ is called the $S$-matrix. It is used to obtain the numbers

$$
N_{a b}{ }^{c}=\sum_{l=0}^{\alpha^{2}-1} \frac{S_{a l} S_{b l} \bar{S}_{l c}}{S_{0 l}}=\frac{1}{\sqrt{\alpha^{2}}} \sum_{l=0}^{\alpha^{2}-1} e^{-2 \pi i l(a+b-c)}=\delta_{a+b, c} .
$$

These numbers allow us to provide the space of characters with a product structure, which we denote by $\times_{V}$, as follows:

$$
\chi_{M_{\alpha, a}}(u ; \tau) \times_{V} \chi_{M_{\alpha, b}}(u ; \tau):=\sum_{c=0}^{\alpha^{2}-1} N_{a b}{ }^{c} \chi_{M_{\alpha, c}}(u ; \tau)=\chi_{M_{\alpha, a+b}}(u ; \tau) .
$$

This product coincides with the Grothendieck ring of characters, that is, the ring structure on the space of characters induced by the fusion product of modules

$$
\chi_{M_{\alpha, a}}(u ; \tau) \times_{G} \chi_{M_{\alpha, b}}(u ; \tau):=\chi_{M_{\alpha, a} \times_{f} M_{\alpha, b}}(u ; \tau)=\chi_{M_{\alpha, a+b}}(u ; \tau) .
$$

The Verlinde formula predicts that the ring of characters obtained from the modular transformations of characters is the Grothendieck ring of characters [V]. In the case of rational conformal field theories this was proven by Huang $\underline{\mathrm{H}}$.

Here we are interested in non-rational conformal field theories that contain a set of uncountable size of modules of the chiral algebra and that are quasi-rational in the sense that the fusion product of two indecomposable modules is a finite sum of indecomposable modules. Then one needs to establish the modularity of the characters and understand how these can be used to define a product structure on the space of characters before postulating an analogue of the Verlinde formula. There are examples of this type that are well understood, namely the case of $\widehat{\mathfrak{g l}}(1 \mid 1)$ CQS and the case of an admissible but non-integer level $\widehat{\mathfrak{s l}}(2)$ theory CR3. In the first example characters are expressed in terms of Jacobi theta functions, while in the second characters are often divergent sums and need to be interpreted as distributions.

We use the modular transformations of characters (Theorem 4.4) to compute a product on the $\mathbb{C}$-span of characters. The fusion product of $\widehat{\mathfrak{g l}}(1 \mid 1)$ is known and the fusion multiplicities are at most one [CR1]. Since the operator product algebra of $\widehat{\mathfrak{g l}}(1 \mid 1)$-modules [SS, $\mathrm{CR} \varnothing$ is also known, it is clear whether there exist intertwining operators between modules of the extended algebra. However, their multiplicities remain unknown. In analogy to $\widehat{\mathfrak{g l}}(1 \mid 1)$ it seems reasonable that these multiplicities are either zero or one. The comparison with the fusion product gives

Theorem 1.2. The product on the space of characters obtained from the modular transformations coincides with the product of the Grothendieck ring of characters if and only if the fusion multiplicities of the extended algebra are at most one.

In contrast to the situation in rational conformal field theories, we have to deal with two subtleties. The modular $S$-transformation involves characters whose weight-labels are not necessarily real. We analytically continue them to real weightlabels. Furthermore, non-generic modules are quotients of reducible generic modules implying that there are linear dependencies between characters of non-generic and generic modules. Using a regularization scheme we use these to express the characters of non-generic modules in terms of generic ones. 
Constructions of this type have been used before. In the cases of $\widehat{\mathfrak{g l}}(1 \mid 1)$ and admissible level $\widehat{\mathfrak{s l}}(2)$, similar to our example, the computation of the ring of characters from the modular transformations involves the interpretation of characters of non-generic modules as infinite sums of characters of generic ones. Further, in the case of $\widehat{\mathfrak{g l}}(1 \mid 1)$ a similar analytic continuation as in our example has been performed.

This work is organized as follows. In Section 2, we summarize the results needed on extended algebras of $\widehat{\mathfrak{g l}}(1 \mid 1)$ of [CR1]. In Section 3, following Zwegers' work, we introduce higher-level Appell-Lerch sums and compute their modular transformation properties. We also introduce a variant of the Mordell integral. In Section 4, we compute the modular transformations of the characters of the modules of the extended algebras. Especially, we give a representation theoretic meaning for the Mordell integral and we find that the $\mathbb{C}$-span of certain finite sets of characters of non-generic modules combined with sets of uncountable size of generic characters are representations of $\mathrm{SL}(2 ; \mathbb{Z})$. In Section 5 , we use the modular transformations to define a product structure on the space of characters and compare it with the Grothendieck ring of characters as far as we know the latter.

\section{Extensions of the Affine Lie superalgebra $\widehat{\mathfrak{g l}}(1 \mid 1)$}

In this section, we summarize results of the affine Lie superalgebra $\widehat{\mathfrak{g l}}(1 \mid 1)$ and the extended algebras of $\widehat{\mathfrak{g l}}(1 \mid 1)$ discussed in [CR1.

The affine Lie superalgebra $\widehat{\mathfrak{g l}}(1 \mid 1)$ is an infinite dimensional Lie superalgebra with generators $E_{n}, N_{n}, \Psi_{n}^{ \pm}(n$ in $\mathbb{Z})$ and $K, d$, where $K$ is central and $d$ is a derivation. The $\Psi_{n}^{ \pm}$are odd, while all others are even elements of the superalgebra. The non-vanishing relations of the remaining generators are

$$
\left[E_{n}, N_{m}\right]=K n \delta_{n+m, 0}, \quad\left[N_{n}, \Psi_{m}^{ \pm}\right]=\Psi_{n+m}^{ \pm}, \quad\left\{\Psi_{n}^{-}, \Psi_{m}^{+}\right\}=E_{n+m}+K n \delta_{n+m, 0} .
$$

One usually considers representations with fixed $K$-eigenvalue $k \in \mathbb{R}$. Using the standard Sugawara construction $[\mathbf{S}$, there is a copy of the Virasoro algebra of central charge zero with generators

$$
\begin{aligned}
L_{n}= & \frac{1}{2 k}\left(2 N_{n} E_{0}-E_{n}+\Psi_{n}^{-} \Psi_{0}^{+}+\Psi_{0}^{-} \Psi_{n}^{+}+\frac{1}{k} E_{n} E_{0}\right) \\
& +\frac{1}{k} \sum_{m>0}\left(E_{n-m} N_{m}+N_{n-m} E_{m}+\Psi_{n-m}^{-} \Psi_{m}^{+}-\Psi_{n-m}^{+} \Psi_{m}^{-}+\frac{1}{k} E_{n-m} E_{m}\right) .
\end{aligned}
$$

We remark that the level can be rescaled so as to normalize it to one, and to simplify notation we set $k=1$ from now on. Let $V$ be a super vector space and $\rho: \widehat{\mathfrak{g l}}(1 \mid 1) \rightarrow \operatorname{End}(V)$ a representation of $\widehat{\mathfrak{g l}}(1 \mid 1)$. We then define its character as

$$
\chi_{\rho}(u, v ; \tau)=\operatorname{str}_{V}\left(q^{\rho\left(L_{0}\right)} z^{\rho\left(N_{0}\right)} y^{\rho\left(E_{0}\right)}\right),
$$

with $q=e^{2 \pi i \tau}, z=e^{2 \pi i u}$ and $y=e^{2 \pi i v}$. Representations were explicitly constructed in $[\mathrm{SS}, \mathrm{L}, \mathrm{CS}, \mathrm{CR} \varnothing]$ using free field realizations of the affine vertex superalgebra of $\mathfrak{g l}(1 \mid 1)$ and its modules. Irreducible representations fall into two families. Generically Verma modules are irreducible; in this case they are called typical. These are highest-weight representations characterized by the weight label $(e, n), e \notin \mathbb{Z}, n \in \mathbb{C}$. We denote them by $\mathcal{T}_{n, e}$ and we require the highest-weight state to have parity $\lfloor e\rfloor$, 
where the number $\lfloor e\rfloor$ is the largest integer smaller than or equal to the real part of $e$. Then the character is

$$
\chi_{\mathcal{T}_{n, e}}(u, v ; \tau)=i(-1)^{\lfloor e\rfloor} y^{e} z^{n} q^{\Delta_{n, e}} \frac{\theta_{1}(u ; \tau)}{\eta^{3}(\tau)},
$$

where the number $\Delta_{n, e}=n e+e^{2} / 2$ is called the conformal dimension of the highest-weight vector of this module. It is required to be real. Representations of the non-generic family are irreducible quotients of Verma modules and are called atypical. They are characterized by the weight label $(\ell, n), \ell \in \mathbb{Z}, n \in \mathbb{R}$. We denote them by $\mathcal{A}_{n, \ell}$ with character

$$
\chi_{\mathcal{A}_{n, \ell}}(u, v ; \tau)=-(-1)^{\ell} i y^{\ell} \frac{z^{n+1 / 2} q^{\Delta_{n, \ell}}}{1-z q^{\ell}} \frac{\theta_{1}(u ; \tau)}{\eta^{3}(\tau)} \times\left\{\begin{array}{cl}
z^{-1 / 2} & \text { if } \ell>0 \\
1 & \text { if } \ell=0 \\
z^{1 / 2} q^{\ell} & \text { if } \ell<0
\end{array}\right.
$$

Here we choose the parity of highest-weight states to agree with the parity given by the free field realizations of $\mathrm{CR} 2, \mathrm{CR} \varnothing$. Remark that the vacuum module of the affine vertex algebra of $\mathfrak{g l}(1 \mid 1)$ is $\mathcal{A}_{0,0}$. This algebra can be extended by atypical modules. For this, note that the fusion product of atypical modules is

$$
\mathcal{A}_{n, \ell} \times \mathcal{A}_{n^{\prime}, \ell^{\prime}}=\mathcal{A}_{n+n^{\prime}-\epsilon\left(\ell, \ell^{\prime}\right), \ell+\ell^{\prime}},
$$

with $\epsilon\left(\ell, \ell^{\prime}\right)=\epsilon(\ell)+\epsilon\left(\ell^{\prime}\right)-\epsilon\left(\ell+\ell^{\prime}\right)$ and $\epsilon(\ell)$ being $1 / 2,0$ or $-1 / 2$ according to whether $\ell \in \mathbb{Z}$ is positive, zero or negative, respectively. The fusion rules have been computed in CR1, and the parity of the highest-weight state can be read off from the explicit free field realization of the algebra and its modules [CR2, CR $\varnothing$. The extended algebras found in CR1 are of the following type. Let $\ell$ be a positive integer and $n$ such that

$$
\Delta_{n+1 / 2, \ell}=n \ell+\frac{\ell(\ell+1)}{2} \in \frac{1}{2} \mathbb{Z}_{>0} .
$$

Then the fusion orbit of $\mathcal{A}_{n+1 / 2, \ell}$ and its conjugate $\mathcal{A}_{-n-1 / 2,-\ell}$ is

$$
\mathrm{A}_{0,0}^{n, \ell}=\bigoplus_{m \in \mathbb{Z}} \mathcal{A}_{m n+\epsilon(m \ell), m \ell}
$$

This is the vacuum module of a $\mathrm{W}$-algebra, let us denote it by $\mathfrak{W}_{n, \ell}$, extending $\widehat{\mathfrak{g l}}(1 \mid 1)$. We require that $a=n / \ell \in \mathbb{Z}$. Let $0<\ell^{\prime}<\ell$ and $m, m^{\prime} \in \mathbb{Z}$. Then (2.2) implies that

$$
\mathrm{A}_{n^{\prime}, \ell^{\prime}}^{n, \ell}=\bigoplus_{m \in \mathbb{Z}} \mathcal{A}_{\left(m \ell+\ell^{\prime}\right) a+n^{\prime}+\epsilon\left(m \ell+\ell^{\prime}\right), m \ell+\ell^{\prime}}
$$

is an irreducible module for $\mathfrak{W}_{n, \ell}$. We call it atypical as it is composed of atypical irreducible $\widehat{\mathfrak{g l}}(1 \mid 1)$ modules. Its character can be read from (2.1) and equals

$$
\chi_{\mathrm{A}_{n^{\prime}, \ell^{\prime}}^{n, \ell}}(u, v ; \tau)=-i \frac{\theta_{1}(u ; \tau)}{\eta^{3}(\tau)} \sum_{m \in \mathbb{Z}} \frac{\left(-y z^{a}\right)^{m \ell+\ell^{\prime}} z^{n^{\prime}+\frac{1}{2}} q^{\frac{1}{2}\left(m \ell+\ell^{\prime}\right)\left(\left(m \ell+\ell^{\prime}\right)(2 a+1)+2 n^{\prime}+1\right)}}{1-z q^{m \ell+\ell^{\prime}}} .
$$

Next, we combine generic $\widehat{\mathfrak{g l}}(1 \mid 1)$ modules into irreducible modules of $\mathfrak{W}_{n, \ell}$. The fusion rules for atypical-typical $\widehat{\mathfrak{g l}}(1 \mid 1)$ modules are

$$
\mathcal{A}_{n, \ell} \times \mathcal{T}_{n^{\prime}, e^{\prime}}=\mathcal{T}_{n+n^{\prime}-\epsilon(\ell), \ell+e^{\prime}} .
$$


This implies that the direct sum of typical modules

$$
\mathrm{T}_{n^{\prime}, e^{\prime}}^{n, \ell}=\bigoplus_{m \in \mathbb{Z}} \mathcal{T}_{m n+n^{\prime}, m \ell+e^{\prime}}
$$

is an irreducible module of $\mathfrak{W}_{n, \ell}$ (for $e^{\prime} \notin \mathbb{Z}$ ); we call it typical. Its character is

$$
\begin{aligned}
& \chi_{\mathrm{T}_{\mathrm{T}^{\prime}, e^{\prime}}^{n, \ell}}(u, v ; \tau)=(-1)^{\left\lfloor e^{\prime}\right\rfloor+\frac{(\ell-1)}{2}} \frac{\theta_{1}(u ; \tau)}{\eta^{3}(\tau)} y^{e^{\prime}-\frac{\ell}{2}} z^{n^{\prime}-\frac{n}{2}} q^{\frac{1}{2}\left(2 n^{\prime} e^{\prime}+e^{\prime 2}-n e^{\prime}-n^{\prime} \ell-\ell e^{\prime}+\frac{\Delta_{n, \ell}}{2}\right)} \\
& (2.3) \quad \times \theta_{1}\left(\frac{\ell-1}{2}+u n+\ell v+\tau\left(n e^{\prime}+n^{\prime} \ell+e^{\prime} \ell-\Delta_{n, \ell}\right) ; 2 \Delta_{n, \ell} \tau\right) .
\end{aligned}
$$

Note, that modules are related as

$$
\mathrm{A}_{n^{\prime}, \ell^{\prime}}^{n, \ell}=\mathrm{A}_{n^{\prime}, \ell^{\prime}+\ell}^{n, \ell} \quad \text { and } \quad \mathrm{T}_{n^{\prime}, e^{\prime}}^{n, \ell}=\mathrm{T}_{n^{\prime}+n, e^{\prime}+\ell}^{n, \ell} .
$$

Typical and atypical characters are related as follows:

$$
\chi_{\mathrm{A}_{n^{\prime}+1, \ell^{\prime}}^{n, \ell}}(u, v ; \tau)-\chi_{\mathrm{A}_{n^{\prime}, \ell^{\prime}}^{n, \ell}}(u, v ; \tau)=\chi_{\mathrm{T}_{n^{\prime}+\ell^{\prime} n / \ell+1 / 2, \ell^{\prime}}^{n, \ell}}(u, v ; \tau) .
$$

This equation has to be taken with care, as the modules $\mathrm{T}_{n^{\prime}, e^{\prime}}^{n, \ell}$ are irreducible if and only if $e^{\prime} \notin \mathbb{Z}$. The characters of $T_{n^{\prime}, \ell^{\prime}}^{n, \ell}$ with $\ell^{\prime} \in \mathbb{Z}$ are characters of reducible but indecomposable modules.

The fusion rules are inherited from those of $\widehat{\mathfrak{g l}}(1 \mid 1)$ up to multiplicities. This means that we know which fusion coefficients are non-zero, but we do not know their precise multiplicities. We conjecture them to be as simple as possible.

Conjecture 2.1. The fusion rules of the extended algebra $\mathfrak{W}_{n, \ell}$ are

$$
\begin{aligned}
& \mathrm{A}_{n^{\prime}, \ell^{\prime}}^{n, \ell} \times_{f} \quad \mathrm{~A}_{n^{\prime \prime}, \ell^{\prime \prime}}^{n, \ell}=\mathrm{A}_{n^{\prime}+n^{\prime \prime}, \ell^{\prime}+\ell^{\prime \prime}}^{n, \ell}, \\
& \mathrm{A}_{n^{\prime}, \ell^{\prime}}^{n, \ell} \times_{f} \quad \mathrm{~T}_{n^{\prime \prime}, e^{\prime \prime}}^{n, \ell}=\mathrm{T}_{n^{\prime}+n^{\prime \prime}+a \ell^{\prime}, \ell^{\prime}+e^{\prime \prime}}^{n, \ell}
\end{aligned}
$$

\section{Appell-Lerch Sums And the Mordell integral}

We study higher-level Appell-Lerch sums and the Mordell integral. First we recall important work of Zwegers [Z2 on (level one) Appell-Lerch sums.

For $u \in \mathbb{C}$ and $\tau \in \mathbb{H}$ we let as before

$$
\theta_{1}(u ; \tau):=-i \sum_{n \in \mathbb{Z}}(-1)^{n} e^{\pi i\left(n+\frac{1}{2}\right)^{2} \tau+2 \pi i u\left(n+\frac{1}{2}\right)} .
$$

For $u, v \in \mathbb{C} \backslash(\mathbb{Z} \tau+\mathbb{Z})$ and $\tau \in \mathbb{H}$ Zwegers defines the normalized Appell-Lerch sum

$$
\mu(u, v ; \tau):=-\frac{e^{2 \pi i u}}{\theta_{1}(v ; \tau)} \sum_{n \in \mathbb{Z}} \frac{(-1)^{n} e^{\pi i \tau n(n+1)} e^{2 \pi i n v}}{1-e^{2 \pi i n \tau+2 \pi i u}} .
$$

Here we consider $A_{1}(u, v ; \tau):=-\theta_{1}(v ; \tau) \mu(u, v ; \tau)$. In order to consider its modular transformation properties we introduce the Mordell integral

$$
h(u ; \tau):=\int_{\mathbb{R}} \frac{e^{\pi i \tau x^{2}-2 \pi z x}}{\cosh \pi x} d x .
$$


Then we have (see Proposition 1.4 in [Z2])

Proposition 3.1. Assume the notation as above. Then

$$
\begin{aligned}
\theta_{1}(u ; \tau+1) & =e^{\frac{\pi i}{4}} \theta_{1}(u ; \tau), \\
\theta_{1}\left(\frac{u}{\tau} ;-\frac{1}{\tau}\right) & =-i \sqrt{-i \tau} e^{\pi i u^{2} / \tau} \theta_{1}(u ; \tau) .
\end{aligned}
$$

For $A_{1}(u, v ; \tau)$ we obtain the following modular transformation properties:

$$
\begin{aligned}
A_{1}(u, v ; \tau+1) & =A_{1}(u, v ; \tau), \\
A_{1}\left(\frac{u}{\tau}, \frac{v}{\tau} ;-\frac{1}{\tau}\right) & =\tau e^{-\pi i\left(u^{2}-2 u v\right) / \tau}\left(A_{1}(u, v ; \tau)+\frac{i}{2} \theta_{1}(v ; \tau) h(u-v ; \tau)\right) .
\end{aligned}
$$

For $K \in \mathbb{N}$ the level $K$ Appell-Lerch sum is defined as

$$
A_{K}(u, v ; \tau)=z^{K / 2} \sum_{n \in \mathbb{Z}} \frac{(-1)^{K n} q^{K n(n+1) / 2} y^{n}}{1-z q^{n}} .
$$

This function was first introduced and studied in [STT]; see also [Z3].

Remark 3.2. Note that

$$
\chi_{\mathrm{A}_{n^{\prime}, 0}^{a, 1}}(u, v ; \tau)=-i \frac{\theta_{1}(u ; \tau)}{\eta^{3}(\tau)} z^{n^{\prime}-a} A_{1+2 a}\left(u, v+a u+\tau\left(n^{\prime}-a\right) ; \tau\right) .
$$

The higher level Appell-Lerch sum can be expressed in terms of the level one sum as follows (also see [Z3]).

Proposition 3.3. The level $K$ Appell-Lerch sum satisfies

$$
A_{K}(u, v ; \tau)=\sum_{m=0}^{K-1} z^{m} A_{1}\left(K u, v+m \tau+\frac{K-1}{2} ; K \tau\right)
$$

and

$$
A_{K}(u, v ; \tau)=K^{-1} z^{(K-1) / 2} \sum_{m=0}^{K-1} A_{1}\left(u, \frac{v}{K}+\frac{m}{K}+\tau \frac{K-1}{2 K} ; \frac{\tau}{K}\right) .
$$

Proof. This is a straightforward rewriting of the sums.

Corollary 3.4. The modular transformation properties of the level $K$ Appell-Lerch sum are

$$
A_{K}(u, v ; \tau+1)=A_{K}(u, v ; \tau)
$$

and

$$
\begin{aligned}
& A_{K}\left(\frac{u}{\tau}, \frac{v}{\tau} ;-\frac{1}{\tau}\right)=\tau e^{-\pi i\left(K u^{2}-2 v u\right) / \tau}\left(A_{K}(u, v ; \tau)\right. \\
& \left.\quad+\frac{i}{2 K} z^{(K-1) / 2} \sum_{m=0}^{K-1} \theta_{1}\left(\frac{K-1}{2 K} \tau+\frac{v}{K}-\frac{m}{K} ; \frac{\tau}{K}\right) h\left(u-\frac{K-1}{2 K} \tau-\frac{v}{K}+\frac{m}{K} ; \frac{\tau}{K}\right)\right) .
\end{aligned}
$$

Proof. Combining Proposition 3.3 and the transformation properties of the level one Appell function (Proposition [3.1) directly yields (3.7). The transformation property (3.6) is obvious. 
We define the variant of the Mordell integral

$$
h_{s}(u ; \tau)= \begin{cases}\int_{\mathbb{R}} \frac{q^{x^{2} / 2} z^{i x}}{\cosh \pi(x-i s)} d x & \text { for } 0 \leq|s| \leq 1, s \neq \pm 1 / 2, \\ \int_{\mathbb{R}-i \epsilon} \frac{q^{x^{2} / 2} z^{i x}}{\cosh \pi(x \mp i / 2)} d x & \text { for } s= \pm 1 / 2\end{cases}
$$

where $\epsilon>0$. We compute

$$
\int_{\mathbb{R}+i s} \frac{q^{x^{2} / 2} z^{i x}}{\cosh \pi(x-i s)} d x=q^{-\frac{s^{2}}{2}} z^{-s} \int_{\mathbb{R}} \frac{q^{x^{2} / 2} z^{i x} e^{-2 \pi \tau s x}}{\cosh \pi x} d x=q^{-\frac{s^{2}}{2}} z^{-s} h(u+s \tau ; \tau) .
$$

Hence, we have for $-\frac{1}{2} \leq s<\frac{1}{2}$ that

$$
h_{s}(u ; \tau)-q^{-s^{2} / 2} z^{-s} h(u+s \tau ; \tau)=\left(\int_{\mathbb{R}}-\int_{\mathbb{R}+i s}\right) \frac{q^{x^{2} / 2} z^{i x}}{\cosh \pi(x-i s)} d x=0 .
$$

Proposition 3.5. For $-1 / 2 \leq s<1 / 2$ the Mordell integral has the following transformation property:

$$
h(u+s \tau)=q^{s^{2} / 2} z^{s} h_{s}(u ; \tau) .
$$

We remark that $h_{1}(u ; \tau)=-h(u ; \tau)$. The transformation properties of the Mordell integral for integral $s$ can be found in [Z3].

\section{Transformation Properties of CHARACTERS}

In this section, we compute the modular transformation properties of the characters. For reasons of brevity we omit the elliptic transformations. The idea is to reduce the computation to the family $\ell=1$. We need the following observation.

Lemma 4.1. Let $\xi_{\ell}=e^{2 \pi i / \ell}$ and $I:=\mathbb{Z} \cap[n, n+\ell-1]$; then the atypical characters satisfy

$$
\begin{aligned}
\chi_{\mathrm{A}_{n^{\prime}, t}^{n, \ell}}(u, v ; \tau) & =\frac{1}{\ell} \sum_{s \in I} \xi_{\ell}^{-t s} \chi_{\mathrm{A}_{n^{\prime}, 0}^{a, 1}}\left(u, v+\frac{s}{\ell} ; \tau\right) \text { and } \\
\chi_{\mathrm{A}_{n^{\prime}, 0}^{a, 1}}\left(u, v+\frac{t}{\ell} ; \tau\right) & =\sum_{s \in I} \xi_{\ell}^{t s} \chi_{\mathrm{A}_{n^{\prime}, s}^{n, \ell}}(u, v ; \tau) .
\end{aligned}
$$

The typical ones satisfy

$$
\begin{aligned}
& \chi_{\mathrm{T}_{n^{\prime}+t n / \ell, e^{\prime}+t}^{n, \ell}}(u, v ; \tau)=\frac{1}{\ell} \sum_{s \in I} \xi_{\ell}^{-s t} e^{-2 \pi i e^{\prime} s / \ell} \chi_{\mathrm{T}_{\mathrm{T}^{\prime}, e^{\prime}}^{a, 1}}\left(u, v+\frac{s}{\ell} ; \tau\right) \quad \text { and } \\
& \chi_{\mathrm{T}_{n^{\prime}, e^{\prime}}^{a, 1}}\left(u, v+\frac{s}{\ell} ; \tau\right)=e^{2 \pi i e^{\prime} s / \ell} \sum_{t \in I} \xi_{\ell}^{s t} \chi_{\mathrm{T}_{n^{\prime}+t n / \ell, e^{\prime}+t}^{n, \ell}}(u, v ; \tau)
\end{aligned}
$$

Proof. The statement follows using results on sums over $\ell$-th roots of unity.

Therefore it suffices to deduce the modular transformation properties of $\chi_{\mathrm{A}_{n^{\prime}, 0}^{a, 1}}(u, v+s / \ell ; \tau)$. 
Lemma 4.2. Let $-\ell / 2 \leq s, t<\ell / 2$ and define

$$
a_{m}(x)=\frac{a(a-m)}{2 a+1}+\frac{a}{2}+i x(a+1), \quad e_{m}(x)=\frac{(a-m)}{2 a+1}+\frac{1}{2}-i x
$$

for $x \in \mathbb{R}$. Then

$$
\begin{aligned}
\chi_{\mathrm{A}_{t / \ell, 0}^{a, 1}}\left(\frac{u}{\tau}, \frac{v}{\tau}+\frac{s}{\ell} ;-\frac{1}{\tau}\right)= & e^{2 \pi i u v / \tau}\left[\chi_{\mathrm{A}_{s / \ell, 0}^{a, 1}}\left(u, v-\frac{t}{\ell} ; \tau\right)\right. \\
& \left.+\frac{1}{2} \sum_{m=0}^{2 a} \int_{\mathbb{R}} d x \frac{\chi_{\mathrm{T}_{a_{m-s / \ell}}^{a, 1}(x), e_{m-s / \ell}(x)}\left(u, v-\frac{t}{\ell} ; \tau\right)}{\cosh \left(\pi\left(x+i \frac{a-m+\frac{s}{\ell}}{2 a+1}\right)\right)}\right] .
\end{aligned}
$$

Proof. We let $K:=2 a+1$. By (3.3) we see that

$$
\begin{aligned}
\chi_{\mathrm{A}_{t / \ell, 0}^{a, 1}}\left(\frac{u}{\tau}, \frac{v}{\tau}+\frac{s}{\ell} ;-\frac{1}{\tau}\right)= & e^{2 \pi i u v / \tau} \chi_{\mathrm{A}_{s / \ell, 0}^{a, 1}}\left(u, v-\frac{t}{\ell} ; \tau\right) \\
& +-\frac{1}{2} \frac{\theta_{1}(u ; \tau)}{\eta^{3}(\tau)} e^{2 \pi i u v / \tau} z^{\frac{s}{\ell}-a} \\
& \times \sum_{m=0}^{2 a} z^{m} \theta_{1}\left(v+a u+\tau\left(\frac{s}{\ell}-a+m\right)-\frac{t s}{\ell} ; K \tau\right) \\
& \times h\left(u(1+a)-v-\tau\left(\frac{s}{\ell}-a+m\right)+\frac{t}{\ell} ; K \tau\right) .
\end{aligned}
$$

Using Proposition 3.5 we can rewrite the latter expression as the sum of integrals over the typical characters for the given parameters.

We compute the modular properties of the typical characters. More specifically, we are interested in

$\chi_{\mathrm{T}_{a_{m-\frac{s}{\ell}}^{a, 1}(x), e_{m-\frac{s}{\ell}}(x)}}(u, v ; \tau)=\frac{\theta_{1}(u ; \tau)}{\eta^{3}(\tau)} y^{b-i x} z^{a b+(a+1) i x} q^{\frac{K}{2}\left(x^{2}+b^{2}\right)} \theta_{1}(v+u a+K b \tau ; K \tau)$

with parameters $b=(a-m+s / \ell) / K$ and $K=2 a+1$. We have

Lemma 4.3. Let $0 \leq m \leq 2 a$ and $-\frac{\ell}{2} \leq s \leq \frac{\ell}{2}$. Then the typical character satisfies

$$
\begin{aligned}
\chi_{\mathrm{T}_{a_{m-\frac{s}{\ell}}(x), e_{m-\frac{s}{\ell}}(x)}^{a, 1}}\left(\frac{u}{\tau}, \frac{v}{\tau}+\frac{t}{\ell} ;-\frac{1}{\tau}\right) \\
=e^{2 \pi i u v / \tau} \sum_{m^{\prime}=0}^{K-1} \int_{\mathbb{R}} d w e^{2 \pi i A_{m^{\prime}}(w)} \chi_{\mathrm{T}_{a_{m^{\prime}-\frac{t}{\ell}}^{a, 1}(w), e_{m^{\prime}-\frac{t}{\ell}}(w)}}\left(u, v-\frac{s}{\ell} ; \tau\right),
\end{aligned}
$$

where $A_{m^{\prime}}(w)=-\frac{i x t}{\ell}-\frac{i w s}{\ell}-w K x+\frac{s t}{\ell^{2} K}-\frac{\left(m^{\prime}+1 / 2\right)(m 1 / 2)}{K}+\frac{1}{4}$.

Proof. Let $\tilde{y}:=e^{2 \pi i v / \tau}, \tilde{z}:=e^{2 \pi i u / \tau}$, and $\tilde{q}:=e^{-2 \pi i / \tau}$. We compute

$$
\begin{aligned}
\chi_{\mathrm{T}_{a_{m-s}^{a, 1}(\ell), e} m-s / \ell}(x) & \left(\frac{u}{\tau}, \frac{v}{\tau}+\frac{t}{\ell} ;-\frac{1}{\tau}\right)=\frac{i}{\tau} \frac{\theta_{1}(u ; \tau)}{\eta^{3}(\tau)} \tilde{y}^{-i x} \tilde{z}^{i x(a+1)} \tilde{q}^{\frac{K}{2} x^{2}} e^{\frac{\pi i}{K \tau}(v+u a)^{2}} \\
& \times e^{\frac{\pi i}{\tau} u^{2}} e^{2 \pi x t / \ell} z^{\frac{a t}{\ell K}} y^{\frac{t}{\ell K}} q^{\frac{t^{2}}{2 K \ell^{2}}} e^{-\pi i K u^{\prime 2} / \tau} \theta_{1}\left(\frac{K}{\tau} u^{\prime} ;-\frac{K}{\tau}\right)
\end{aligned}
$$


with $u^{\prime}=\frac{v}{K}+\frac{u a}{K}-b+\frac{t \tau}{K \ell}$. The standard Gauss integral gives

$$
\frac{1}{\sqrt{-i \tau K}} \tilde{y}^{-i x} \tilde{z}^{i x(a+1)} \tilde{q}^{\frac{K}{2} x^{2}}=e^{-\frac{\pi i}{\tau K}(v-(a+1) u)^{2}} \int_{\mathbb{R}} d w q^{\frac{K}{2} w^{2}} z^{i w(a+1)} y^{-i w} e^{-2 \pi i w K x} .
$$

Together with

$$
\begin{aligned}
\theta_{1}\left(u ; \frac{\tau}{K}\right)=\sum_{n=0}^{K-1} q^{(n-(K-1) / 2)^{2} / 2 K} e^{2 \pi i(n-(K-1) / 2)(u+1 / 2)} & \\
& \times \theta_{1}\left(K u+\tau\left(n-\frac{K-1}{2}\right)+\frac{K-1}{2} ; K \tau\right)
\end{aligned}
$$

this implies

$$
\begin{aligned}
& \chi_{\mathrm{T}_{a_{m-\frac{s}{\ell}}(x), e_{m-\frac{s}{\ell}}(x)}}\left(\frac{u}{\tau}, \frac{v}{\tau}+\frac{t}{\ell} ;-\frac{1}{\tau}\right) \\
& =e^{2 \pi i \frac{u v}{\tau}} \sum_{m^{\prime}=0}^{K-1} \int_{\mathbb{R}} d w e^{2 \pi i A_{m^{\prime}}(w)} \chi_{\mathrm{T}_{a_{m^{\prime}-\frac{t}{\ell}}^{a, 1}(w), e_{m^{\prime}-\frac{t}{\ell}}(w)}}\left(u, v-\frac{s}{\ell} ; \tau\right),
\end{aligned}
$$

where we changed summation to $m^{\prime}=2 a-n$ in (4.3).

Define the sets

$$
\begin{gathered}
S=\{s \in \mathbb{Z} \mid-\ell / 2 \leq s<\ell / 2\}, \\
M=\left\{m \in \frac{1}{\ell} \mathbb{Z} \mid-\frac{\ell}{2}(2 a+1)<m \leq \frac{\ell}{2}(2 a+1)\right\} .
\end{gathered}
$$

Theorem 4.4. Let $s, s^{\prime}, t, t^{\prime} \in S, r, c \in M, x \in \mathbb{R}$ and define the S-matrices

$$
\begin{aligned}
S_{\left(t / \ell, t^{\prime}\right)}^{\left(s / \ell, s^{\prime}\right)} & =\frac{1}{\ell} \xi_{\ell}^{-t^{\prime} s-s^{\prime} t}, \\
S_{\left(t / \ell, t^{\prime}\right)}^{\left(a_{r}(x), e_{r}(x)\right)} & =\frac{1}{2 \ell} \frac{e^{2 \pi i\left(t^{\prime} r-e_{r}(x) t / \ell\right)}}{\sin \left(\pi e_{r}(x)\right)}, \\
S_{\left(a_{r}(x), e_{r}(x)\right)}^{\left(a_{c}(w) e_{c}(w)\right)} & =\frac{1}{\ell}(-1)^{\left\lfloor e_{r}(x)\right\rfloor+\left\lfloor e_{c}(w)\right\rfloor} \\
& \times e^{2 \pi i\left(e_{r}(x) K e_{c}(w)+e_{r}(x)\left(c-2 a-\frac{1}{2}\right)+e_{c}(w)\left(r-2 a-\frac{1}{2}\right)+\frac{1}{4}\right)} .
\end{aligned}
$$

Then we have the following modular transformation properties:

$$
\begin{aligned}
\chi_{\mathrm{A}_{t / \ell, t^{\prime}}^{n, \ell}}\left(\frac{u}{\tau}, \frac{v}{\tau} ;-\frac{1}{\tau}\right)= & e^{2 \pi i u v / \tau}\left[\sum_{s, s^{\prime} \in S} S_{\left(t / \ell, t^{\prime}\right)}^{\left(s / \ell, s^{\prime}\right)} \chi_{\mathrm{A}_{s / \ell, s^{\prime}}^{n, \ell}}(u, v ; \tau)\right. \\
& \left.+\sum_{r \in M} \int_{\mathbb{R}} d x S_{\left(t / \ell, t^{\prime}\right)}^{\left(a_{r}(x), e_{r}(x)\right)} \chi_{\mathrm{T}_{a_{r}(x), e_{r}(x)}^{n, \ell}}(u, v ; \tau)\right], \\
\chi_{\mathrm{A}_{t / \ell, t^{\prime}}^{n, \ell}}(u, v ; \tau+1)= & \xi_{\ell}^{t t^{\prime}} \chi_{\mathrm{A}_{t / \ell, t^{\prime}}^{n, \ell}}(u, v ; \tau), \\
\chi_{\mathrm{T}_{a_{r}(x), e_{r}(x)}^{n, \ell}}\left(\frac{u}{\tau}, \frac{v}{\tau} ;-\frac{1}{\tau}\right)= & e^{2 \pi i u v / \tau} \sum_{c \in M} \int_{\mathbb{R}} d w S_{\left(a_{r}(x), e_{r}(x)\right)}^{\left(a_{c}(w), e_{c}(w)\right)} \chi_{\mathrm{T}_{a_{c}(w), e_{c}(w)}^{n, \ell}}(u, v ; \tau), \\
\chi_{\mathrm{T}_{a_{r}(x), e_{r}(x)}^{n, \ell}}(u, v ; \tau+1)= & e^{\pi i\left(\frac{(a-r)^{2}}{K^{2}}+(a-r)+K x^{2}+\frac{a}{2}+\frac{1}{4}\right)} \chi_{\mathrm{T}_{a_{r}(x), e_{r}(x)}^{n, \ell}}(u, v ; \tau) .
\end{aligned}
$$


Proof. The formulas for the $T$-transformations follow directly. For the formula for $S$-transformations we first employ Lemma 4.1 and then use Lemma 4.2 respectively Lemma 4.3. The "reverse" application of Lemma 4.1 yields the results where we use the periodicity of cosh and change the summation index to $r:=m-s / \ell-s^{\prime} K$ for the formula for $\chi_{\mathrm{A}_{t / \ell, t^{\prime}}^{n, \ell}}\left(\frac{u}{\tau}, \frac{v}{\tau} ;-\frac{1}{\tau}\right)$. For $\chi_{\mathrm{T}_{a_{r}(x), e_{r}(x)}^{n, \ell}}\left(\frac{u}{\tau}, \frac{v}{\tau} ;-\frac{1}{\tau}\right)$ we set $r=m-s / \ell-s^{\prime} K$ and $c=m^{\prime}-t / \ell-t^{\prime} K$. Again, the repeated use of Lemma 4.1 together with a change of the summation index yields the result.

\section{THE RING OF CHARACTERS}

In this section, we explain how to use the mock modular transformation properties in Theorem 4.4 to provide the vector space of characters with a product structure. We show that this product coincides with the corresponding product in the Grothendieck ring of characters if and only if Conjecture 2.1 holds. While the use of the Verlinde formula is straightforward in rational conformal field theories, we have to deal with two subtleties here. Namely, we have to analytically continue the S-matrix and we have to view characters of atypical modules as (regularized) sums of characters of typical ones. Similar techniques were applied in the computation of the Verlinde formula of $\widehat{\mathfrak{g l}}(1 \mid 1)$ [CR1] and a commutant subalgebra of one of our theories [CR3].

We are interested in characters with real weight labels, while the modular transformations of the last section involved not necessarily real weights. Hence, we define the $S$-matrix as an analytic continuation to real weight labels of (4.4). Recall that $a_{r}(x)=2 a-r-e_{r}(x)(a+1)+1 / 2$ as in (4.1).

Definition 5.1. Let $s, s^{\prime}, t, t^{\prime} \in S, r, r^{\prime} \in M, e, e^{\prime} \in \mathbb{R}$, and let $m=2 a-r, m^{\prime}=$ $2 a-r^{\prime}$. Then we define

$$
\begin{aligned}
S_{a a\left(t / \ell, t^{\prime}\right)}^{\left(s / \ell, s^{\prime}\right)} & =\frac{1}{\ell} \xi_{\ell}^{-t^{\prime} s-s^{\prime} t}, \\
S_{a t}^{\left(m-\left(t / \ell, t^{\prime}\right)\right.} & =\frac{1}{2 \ell} \frac{e^{\left.2 \pi i\left(t^{\prime} m-e t / \ell\right) e+1 / 2, e\right)}}{\sin (\pi e)}, \\
S_{t t}^{\left(m^{\prime}-(a+1) e^{\prime}+1 / 2, e^{\prime}\right)} & =\frac{1}{\ell}(-1)^{\lfloor e\rfloor+\left\lfloor e^{\prime}\right\rfloor} e^{2 \pi i\left(e K e^{\prime}-e\left(m^{\prime}-1 / 2\right)-e^{\prime}(m-1 / 2)+1 / 4\right)} .
\end{aligned}
$$

We call $S_{t t}$ the typical (and $S_{a a}$ the atypical) $S$-matrix.

Proposition 5.2. The typical and atypical S-matrices are symmetric

$$
S_{t t}^{\left(m^{\prime}-(a+1) e^{\prime}+1 / 2, e^{\prime}\right)}=S_{t t}^{(m-(a+1) e+1 / 2, e)}\left(\begin{array}{l}
(m+1) e+1 / 2, e) \\
\left(m^{\prime}-(a+1) e^{\prime}+1 / 2, e^{\prime}\right)
\end{array}, \quad S_{a a\left(t / \ell, t^{\prime}\right)}^{\left(s / \ell, s^{\prime}\right)}=S_{a a\left(s / \ell, s^{\prime}\right)}^{\left(t / \ell, t^{\prime}\right)}\right.
$$

and unitary

$$
\begin{aligned}
\sum_{m^{\prime} \in M} \int d e^{\prime} S_{t t}^{\left(m^{\prime}-(a+1) e^{\prime}+1 / 2, e^{\prime}\right)} \bar{S}_{(m-(a+1) e+1 / 2, e)} \begin{array}{c}
\left(m^{\prime \prime}-(a+1) e^{\prime \prime}+1 / 2, e^{\prime \prime}\right) \\
t\left(m^{\prime}-(a+1) e^{\prime}+1 / 2, e^{\prime}\right)
\end{array} & =\delta_{m, m^{\prime \prime}} \delta\left(e-e^{\prime \prime}\right), \\
\sum_{s^{\prime}, s \in S} S_{a a}^{\left(s / \ell, s^{\prime}\right)} \bar{S}_{a a\left(t / t^{\prime}\right)}^{\left(r / \ell, r^{\prime}\right)} & =\delta_{t, r} \delta_{t^{\prime}, r^{\prime}} .
\end{aligned}
$$

The bar denotes complex conjugation. 
Proof. The first statement on the symmetry is clear. For the second statement observe that

$$
\begin{aligned}
& \sum_{m^{\prime} \in M} \int d e^{\prime} S_{t t} \begin{array}{c}
\left(m^{\prime}-(a+1) e^{\prime}+1 / 2, e^{\prime}\right) \\
(m-(a+1) e+1 / 2, e)
\end{array} \\
& (5.1) \quad=\frac{1}{\ell^{2}} \sum_{m^{\prime} \in M} \int d e^{\prime}(-1)^{\left\lfloor m^{\prime}-(a+1) e^{\prime \prime}+1 / 2, e^{\prime \prime}\right)} \\
& \qquad e\rfloor+\left\lfloor e^{\prime \prime}\right\rfloor e^{2 \pi i e^{\prime}\left(e K-e^{\prime \prime} K-m+m^{\prime \prime}\right)} e^{2 \pi i\left(m^{\prime}-1 / 2\right)\left(e^{\prime \prime}-e\right)} .
\end{aligned}
$$

Now

$$
\int d e^{\prime} e^{2 \pi i e^{\prime}\left(e K-e^{\prime \prime} K-m+m^{\prime \prime}\right)}=0
$$

unless $e K-e^{\prime \prime} K=m^{\prime \prime}-m$. So we can consider

$$
\sum_{m^{\prime} \in M} e^{2 \pi i m^{\prime}\left(m-m^{\prime \prime}\right) / K}
$$

which is only non-zero for $m=m^{\prime \prime}$. Then the delta distribution can only be nonzero if $e-e^{\prime} \in \mathbb{Z}$, in which case the phase $e^{\pi i\left(e-e^{\prime}\right)}(-1)^{\lfloor e\rfloor+\left\lfloor e^{\prime}\right\rfloor}$ becomes one. So (5.1) equals $\delta_{m, m^{\prime \prime}} \delta\left(e-e^{\prime \prime}\right)$. Moreover, we have

$$
\sum_{s^{\prime}, s \in S} S_{a a\left(t / \ell, t^{\prime}\right)}^{\left(s / \ell, s^{\prime}\right)} \bar{S}_{a a\left(s / \ell, s^{\prime}\right)}^{\left(r / \ell, r^{\prime}\right)}=\frac{1}{\ell^{2}} \sum_{s^{\prime}, s \in S} \xi_{\ell}^{-t^{\prime} s-s^{\prime} t} \bar{\xi}_{\ell}^{-s^{\prime} r-r^{\prime} s} .
$$

Using standard results on roots of unity we see that this equals $\delta_{t, r} \delta_{t^{\prime}, r^{\prime}}$.

Definition 5.3. Define the structure constants for $t, t^{\prime} \in S, e, e^{\prime} \in \mathbb{R}$, and $m, m^{\prime} \in$ $M$ as

$$
\begin{aligned}
& N_{\left(t / \ell, t^{\prime}\right),(m-(a+1) e+1 / 2, e)}^{\left(m^{\prime}-(a+1) e^{\prime}+1 / 2, e^{\prime}\right)} \\
& \quad=\sum_{k \in M} \int_{\mathbb{R}} d x \frac{S_{a t}^{(k-(a+1) x+1 / 2, x)} S_{t t}^{(k-(a+1) x+1 / 2, x)} \bar{S}_{(m-(a+1) e+1 / 2, e)}^{\left(k-\left(t^{\prime}\right)\right.} \bar{S}_{t t(k-(a+1) x+1 / 2, x)}^{\left(m^{\prime}-(a+1) e^{\prime}+1 / 2, e^{\prime}\right)}}{S_{a t}^{(k-(a+1) x+1 / 2, x)}} .
\end{aligned}
$$

A straightforward computation gives

Proposition 5.4. The structure constants satisfy

$N_{\left(t / \ell, t^{\prime}\right),(m-(a+1) e+1 / 2, e)}^{\left(m^{\prime}-(a+1) e^{\prime}+1 / 2, e^{\prime}\right)}=\delta\left(e-e^{\prime}+\left(m^{\prime}-m-t / \ell\right) / K\right) \delta_{m^{\prime} \equiv m+t / \ell+t^{\prime} K} \quad(\bmod \ell K)$.

Proof. The statement follows from the following computation:

$$
\begin{aligned}
& N_{\left(t / \ell, t^{\prime}\right),(m-(a+1) e+1 / 2, e)}^{\left(m^{\prime}-(a+1) e^{\prime}+1 / 2, e^{\prime}\right)} \\
& \quad=\frac{1}{\ell^{2}} \sum_{k \in M} \int_{\mathbb{R}} d x e^{2 \pi i x K\left(e-e^{\prime}+\left(m^{\prime}-m-t / \ell\right) / K\right)} e^{2 \pi i k\left(e^{\prime}-e-t^{\prime}\right)} e^{\pi i\left(e-e^{\prime}\right)}(-1)^{\lfloor e\rfloor+\left\lfloor e^{\prime}\right\rfloor} \\
& =\frac{1}{K \ell^{2}} \sum_{k \in M} \delta\left(e-e^{\prime}+\left(m^{\prime}-m-t / \ell\right) / K\right) e^{2 \pi i k\left(e^{\prime}-e-t^{\prime}\right)} e^{\pi i\left(e-e^{\prime}\right)}(-1)^{\lfloor e\rfloor+\left\lfloor e^{\prime}\right\rfloor} \\
& =\frac{1}{K \ell^{2}} \sum_{k \in M} \delta\left(e-e^{\prime}+\left(m^{\prime}-m-t / \ell\right) / K\right) e^{2 \pi i \frac{k}{K}\left(m^{\prime}-m-t^{\prime} K\right)} e^{\pi i\left(e-e^{\prime}\right)}(-1)^{\lfloor e\rfloor+\left\lfloor e^{\prime}\right\rfloor} \\
& =\delta\left(e-e^{\prime}+\left(m^{\prime}-m\right) / K\right) \delta_{m^{\prime} \equiv m+t / \ell+t^{\prime} K}(\bmod \ell K) .
\end{aligned}
$$

The delta distribution can only be non-zero if $e-e^{\prime} \in \mathbb{Z}$, in which case the phase $e^{\pi i\left(e-e^{\prime}\right)}(-1)^{\lfloor e\rfloor+\left\lfloor e^{\prime}\right\rfloor}$ becomes one. 
Since $m, m^{\prime} \in M$ and $t, t^{\prime} \in S$, we may choose an $\epsilon \in\{-1,0,1\}$ such that $m+t / \ell+t^{\prime} K+\epsilon \ell K \in M$. We now define and compute an action of atypical characters on typical ones by

$$
\begin{aligned}
& \chi_{\mathrm{A}_{t / \ell, t^{\prime}}^{n, \ell}} \times \chi_{\mathrm{T}_{m-(a+1) e, e}^{n, \ell}}=\sum_{m^{\prime} \in M} \int_{\mathbb{R}} d e^{\prime} N_{\left(t / \ell, t^{\prime}\right),(m-(a+1) e+1 / 2, e)}^{\left(m^{\prime}-(a+1) e^{\prime}+1 / 2, e^{\prime}\right)} \chi_{\mathrm{T}_{m^{\prime}-(a+1) e^{\prime}, e^{\prime}}^{n, \ell}} \\
& =\chi_{\mathrm{T}_{m+t / \ell-(a+1) e+t^{\prime} a+\epsilon \ell a, e+t^{\prime}+\epsilon \ell}^{n, \ell}}=\chi_{\mathrm{T}_{m+t / \ell-(a+1) e+t^{\prime} a, e+t^{\prime}}^{n, \ell}} .
\end{aligned}
$$

For the last equality we used $a \ell=n$ and (2.4). Remark that this action extends to an action of the $\mathbb{C}$-span of atypical characters on the $\mathbb{C}$-span of typical characters by linearity. In order to obtain a product structure on atypical characters we use the relation to typical ones (2.5), namely

$$
\chi_{\mathrm{A}_{s / \ell, s^{\prime}}^{n, \ell}}-\chi_{\mathrm{A}_{s / \ell+m+1, s^{\prime}}^{n, \ell}}=\sum_{i=0}^{m} \chi_{\mathrm{T}_{s / \ell+i+s^{\prime} a+1 / 2, s^{\prime}}^{n, \ell}}
$$

for all $m>0, m \in \mathbb{Z}$ and combine this with (5.2) to get

$$
\chi_{\mathrm{A}_{t / \ell, t^{\prime}}^{n, \ell}} \times\left(\chi_{\mathrm{A}_{s / \ell, s^{\prime}}^{n, \ell}}-\chi_{\mathrm{A}_{s / \ell+m+1, s^{\prime}}^{n, \ell}}\right)=\chi_{\mathrm{A}_{(s+t) / \ell, s^{\prime}+t^{\prime}}^{n, \ell}}-\chi_{\mathrm{A}_{(s+t) / \ell+m+1, s^{\prime}+t^{\prime}}^{n, \ell}} .
$$

We would like to take the limit $m \rightarrow \infty$. Here a regularization is needed. For $\epsilon>0$ we let

$$
\chi_{\mathrm{A}_{n^{\prime}, \ell^{\prime}}^{n, \ell}}^{\epsilon}=q^{\epsilon n^{\prime 2}} \chi_{\mathrm{A}_{n^{\prime}, \ell^{\prime}}^{n, \ell}} .
$$

The product of regularized characters

$$
\chi_{\mathrm{A}_{t / \ell, t^{\prime}}^{n, \ell}}^{\epsilon} \times\left(\chi_{\mathrm{A}_{s / \ell, s^{\prime}}^{n, \ell}}^{\epsilon,}-\chi_{\mathrm{A}_{s / \ell+m+1, s^{\prime}}^{n, \ell}}^{\epsilon}\right)=\chi_{\mathrm{A}_{(s+t) / \ell, s^{\prime}+t^{\prime}}^{n, \ell}}^{\epsilon}-\chi_{\mathrm{A}_{(s+t) / \ell+m+1, s^{\prime}+t^{\prime}}^{n, \ell}}^{\epsilon}
$$

agrees with (5.3) in the limit $\epsilon \rightarrow 0$. The regularized character vanishes at infinity

$$
\lim _{n^{\prime} \rightarrow \infty} \chi_{\mathrm{A}_{n^{\prime}, \ell^{\prime}}^{n, \ell}}^{\epsilon}=0 \quad \text { for all } \epsilon>0 .
$$

Hence, in the limit $m \rightarrow \infty$, the regularized product (5.4) of (5.3) becomes

$$
\chi_{\mathrm{A}_{t / \ell, t^{\prime}}^{n, \ell}}^{\epsilon} \times \chi_{\mathrm{A}_{s / \ell, s^{\prime}}^{n, \ell}}^{\epsilon}=\chi_{\mathrm{A}_{(s+t) / \ell, s^{\prime}+t^{\prime}}^{\epsilon, \ell}}^{\epsilon} .
$$

We summarize

Proposition 5.5. For $t, t^{\prime}, s, s^{\prime} \in S, e \in \mathbb{R}, m \in M$ the modular $S$-transformation gives the following products:

$$
\begin{aligned}
\chi_{\mathrm{A}_{t / \ell, t^{\prime}}^{n, \ell}}(u, v ; \tau) \times \chi_{\mathrm{T}_{m-(a+1) e, e}^{n, \ell}}(u, v ; \tau) & =\chi_{\mathrm{T}_{m+t / \ell-(a+1) e+t^{\prime} a, e+t^{\prime}}^{n, \ell}}(u, v ; \tau), \\
\chi_{\mathrm{A}_{t / \ell, t^{\prime}}^{n, \ell}}^{\epsilon}(u, v ; \tau) \times \chi_{\mathrm{A}_{s / \ell, s^{\prime}}^{n, \ell}}^{\epsilon}(u, v ; \tau) & =\chi_{\mathrm{A}_{(s+t) / \ell, s^{\prime}+t^{\prime}}^{n, \ell}}^{\epsilon}(u, v ; \tau) .
\end{aligned}
$$

Comparing with Conjecture 2.1, we observe

Corollary 5.6. Assume that Conjecture 2.1 is correct, i.e. that fusion multiplicities are at most one. Then the product structure from the modular transformations coincides with the corresponding product in the Grothendieck ring of characters:

$$
\begin{aligned}
\chi_{\mathrm{A}_{t / \ell, t^{\prime}}^{n, \ell}}(u, v ; \tau) \times \chi_{\mathrm{T}_{m-(a+1) e, e}^{n, \ell}}(u, v ; \tau) & \left.=\chi_{\left(\mathrm{A}_{t / \ell, t^{\prime}}^{n, \ell} \times f\right.} \mathrm{T}_{m-(a+1) e, e}^{n, \ell}\right) \\
\chi_{\mathrm{A}_{t / \ell, t^{\prime}}^{n, \ell}}^{\epsilon}(u, v ; \tau) \times \chi_{\mathrm{A}_{s / \ell, s^{\prime}}^{n, \ell}}^{\epsilon}(u, v ; \tau) & =\chi_{\left(\mathrm{A}_{t / \ell, t^{\prime}}^{n, \ell} \times_{f} \mathrm{~A}_{s / \ell, s^{\prime}}^{n, \ell}\right)}^{\epsilon, v ; \tau),}(u, v ; \tau) .
\end{aligned}
$$




\section{ACKNOWLEDGMENTS}

The authors thank Kathrin Bringmann, Stephan Ehlen, and David Ridout for helpful comments on an earlier draft of the paper.

\section{REFERENCES}

[BF] Jan Hendrik Bruinier and Jens Funke, On two geometric theta lifts, Duke Math. J. 125 (2004), no. 1, 45-90, DOI 10.1215/S0012-7094-04-12513-8. MR2097357 (2005m:11089)

[BHT] P. Bowcock, M. Hayes, and A. Taormina, Characters of admissible representations of the affine superalgebra $\widehat{\mathrm{sl}}(2 \mid 1 ; \mathbf{C})_{k}$, Nuclear Phys. B 510 (1998), no. 3, 739-763, DOI 10.1016/S0550-3213(97)00542-7. MR1607156 (99f:81074)

[BriFo] K. Bringmann, A. Folsom, Almost harmonic Maass forms and Kac-Wakimoto characters, preprint.

[BriO] Kathrin Bringmann and Ken Ono, Some characters of Kac and Wakimoto and nonholomorphic modular functions, Math. Ann. 345 (2009), no. 3, 547-558, DOI 10.1007/s00208009-0364-2. MR2534107 (2010f:11061)

[CQS] T. Creutzig, T. Quella, and V. Schomerus, Branes in the GL(1|1) WZNW model, Nuclear Phys. B 792 (2008), no. 3, 257-283, DOI 10.1016/j.nuclphysb.2007.09.014. MR2387340 (2009j:81085)

[CR1] T. Creutzig, D. Ridout, Relating the archetypes of logarithmic conformal field theory, arXiv:1107.2135.

[CR2] T. Creutzig, D. Ridout, W-algebras extending affine gl(1|1), arXiv:1111.5049.

[CR3] Thomas Creutzig and David Ridout, Modular data and Verlinde formulae for fractional level WZW models I, Nuclear Phys. B 865 (2012), no. 1, 83-114, DOI 10.1016/j.nuclphysb.2012.07.018. MR2968504

[CRø] Thomas Creutzig and Peter B. Rønne, The GL(1|1)-symplectic fermion correspondence, Nuclear Phys. B 815 (2009), no. 1-2, 95-124, DOI 10.1016/j.nuclphysb.2009.02.013. MR.2513720 (2010e:81213)

[CS] Thomas Creutzig and Volker Schomerus, Boundary correlators in supergroup WZNW models, Nuclear Phys. B 807 (2009), no. 3, 471-494, DOI 10.1016/j.nuclphysb.2008.07.025. MR.2469132(2010a:81207)

[ET] Tohru Eguchi and Anne Taormina, On the unitary representations of $N=2$ and $N=4$ superconformal algebras, Phys. Lett. B 210 (1988), no. 1-2, 125-132, DOI 10.1016/03702693(88)90360-7. MR959503 (89k:17019)

[FS] B. L. Feigin and A. M. Semikhatov, $\mathcal{W}_{n}^{(2)}$ algebras, Nuclear Phys. B 698 (2004), no. 3, 409-449, DOI 10.1016/j.nuclphysb.2004.06.056. MR2092705 (2005h:17047)

[F] Amanda Folsom, Kac-Wakimoto characters and universal mock theta functions, Trans. Amer. Math. Soc. 363 (2011), no. 1, 439-455, DOI 10.1090/S0002-9947-2010-05181-5. MR:2719689 (2011h:11044)

[G] M. R. Gaberdiel, An introduction to conformal field theory, Rept. Prog. Phys. 63 (2000).

[GQS] Gerhard Götz, Thomas Quella, and Volker Schomerus, The WZNW model on PSU(1, 1|2), J. High Energy Phys. 3 (2007), 003, 48 pp. (electronic), DOI 10.1088/11266708/2007/03/003. MR2313894 (2008k:81279)

[H] Yi-Zhi Huang, Vertex operator algebras and the Verlinde conjecture, Commun. Contemp. Math. 10 (2008), no. 1, 103-154, DOI 10.1142/S0219199708002727. MR2387861 (2009e:17056)

[KP] Victor G. Kac and Dale H. Peterson, Infinite-dimensional Lie algebras, theta functions and modular forms, Adv. in Math. 53 (1984), no. 2, 125-264, DOI 10.1016/00018708(84)90032-X. MR750341 (86a:17007)

[KW1] Victor G. Kac and Minoru Wakimoto, Integrable highest weight modules over affine superalgebras and number theory, Lie theory and geometry, Progr. Math., vol. 123, Birkhäuser Boston, Boston, MA, 1994, pp. 415-456. MR1327543 (96j:11056)

[KW2] Victor G. Kac and Minoru Wakimoto, Integrable highest weight modules over affine superalgebras and Appell's function, Comm. Math. Phys. 215 (2001), no. 3, 631-682, DOI 10.1007/s002200000315. MR.1810948 (2001j:17017)

[L] A. LeClair, The gl(1|1) super-current algebra: The role of twist and logarithmic fields, arXiv:0710.2906 [hep-th]. 
[M] Kei Miki, The representation theory of the $\mathrm{SO}(3)$ invariant superconformal algebra, Internat. J. Modern Phys. A 5 (1990), no. 7, 1293-1318, DOI 10.1142/S0217751X90000593. MR 1044465 (91j:17029)

[S] H. Sugawara, A field theory of currents, Phys. Rev. 170 (1968).

[SS] Volker Schomerus and Hubert Saleur, The GL(1|1) WZW-model: from supergeometry to logarithmic CFT, Nuclear Phys. B $\mathbf{7 3 4}$ (2006), no. 3, 221-245, DOI 10.1016/j.nuclphysb.2005.11.013. MR2195505 (2006m:81264)

[STT] A. M. Semikhatov, A. Taormina, and I. Yu. Tipunin, Higher-level Appell functions, modular transformations, and characters, Comm. Math. Phys. 255 (2005), no. 2, 469-512, DOI 10.1007/s00220-004-1280-7. MR2129953(2007j:11051)

[V] E. P. Verlinde, Fusion rules and modular transformations in $2 D$ conformal field theory, Nucl. Phys. B 300 (1988).

[Z] D. Zagier, Ramanujan's mock theta functions and their applications [d'après Zwegers and Bringmann-Ono], Sèminaire Bourbaki, 60eme annèe, 2006-2007, no. 986.

[Z3] S. Zwegers, Multivariable Appell functions, preprint.

[Z1] S. P. Zwegers, Mock $\theta$-functions and real analytic modular forms (English summary), $q$-series with applications to combinatorics, number theory, and physics (Urbana, IL, 2000), Contemp. Math., vol. 291, Amer. Math. Soc., Providence, RI, 2001, pp. 269-277, DOI 10.1090/conm/291/04907. MR1874536(2003f:11061)

[Z2] S. Zwegers, Mock theta functions, Ph.D. thesis, Utrecht.

Fachbereich Mathematik, Technische Universität Darmstadt, 64289 Darmstadt, GerMANY

E-mail address: alfes@mathematik.tu-darmstadt.de

Fachbereich Mathematik, Technische Universität Darmstadt, 64289 Darmstadt, GerMANY

Current address: Department of Mathematical and Statistical Sciences, 632 CAB, University of Alberta, Edmonton, Alberta T6G 2G1, Canada

E-mail address: creutzig@ualberta.ca 